\title{
WHEN A TOTALLY BOUNDED GROUP TOPOLOGY IS THE BOHR TOPOLOGY OF A LCA GROUP
}

\author{
SALVADOR HERNÁNDEZ AND F. JAVIER TRIGOS-ARRIETA
}

\begin{abstract}
We look at the Bohr topology of maximally almost periodic groups (MAP, for short). Among other results, we investigate when a Hausdorff precompact abelian group $(G, \mathrm{w})$ is the Bohr reflection of a locally compact abelian group. Necessary and sufficient conditions are established in terms of the inner properties of w. As an application, an example of a MAP group $(G, t)$ is given such that every closed, metrizable subgroup $N$ of $b G$ with $N \cap G=\{0\}$ preserves compactness but $(G, t)$ does not strongly respect compactness. Thereby, we respond to Questions 4.1 and 4.3 in [6].
\end{abstract}

\section{INTRODUCTION}

For each topological group $G$ (resp. $(G, t)$ if we wish to emphasize the topology considered on $G$ ) there is associated a compact Hausdorff group $b G$ and a continuous homomorphism $b$ from $G$ onto a dense subgroup of $b G$ with the following universal property: for every continuous homomorphism $h$ from $G$ into a compact group $K$ there is a continuous homomorphism $h^{+}$from $b G$ into $K$ such that $h=h^{+} \circ b$. The group $b G$ is essentially unique; it is called the Bohr compactification of $G$ (see Heyer [15] for a careful examination of $b G$ and its properties). Here, we restrict our attention to the abelian groups such that the homomorphism $b$ above is one-to-one; these are exactly the maximally almost periodic (MAP) abelian groups.

For such a topological group $G$ (resp. $(G, t))$ we denote by $G^{+}$(resp. $\left(G, t^{+}\right)$the underlying group of $G$ equipped with its Bohr topology. Evidently, $G^{+}$is an example of a Hausdorff precompact group. The following notion plays an important rôle for the rest of this discussion: A MAP abelian group $G$ is said to respect a topological property $\mathcal{P}$ if a subset $A$ of $G$ has $\mathcal{P}$ as a subspace of $G$ if and only if $A$ has $\mathcal{P}$ as a subspace of $G^{+}$(Trigos-Arrieta [20]).

The question of the disposition or placement of a LCA group $G$ within its Bohr compactification $b G$ has been investigated by many researchers. It is known for such $G$, for example, that $G^{+}$is sequentially closed in $b G$ in the sense that no sequence from $G$ can converge to a point of $b G \backslash G$ [19]. And Glicksberg [11] has shown that LCA groups respect compactness. This result concerning LCA groups is one of the pivotal results of the subject, often referred to as Glicksberg's theorem. Hughes [16] proved a generalization of Glicksberg's theorem to (not necesary Abelian) locally compact groups by considering the weak topology generated by the continuous irreducible unitary group representations. Several authors have achieved additional results which continue the lines of investigation suggested above (see [10]). Nevertheless many questions relating the topology of a MAP group with its Bohr topology are still open in general. In this paper, we continue with the investigation of the Bohr topology of maximally almost periodic groups accomplished in [6] and [8]. Among other results, we investigate when a Hausdorff precompact abelian group $(G, \mathrm{w})$ is the Bohr reflection of a locally compact abelian group. Necessary and sufficient conditions are established in terms of the inner properties of w. As an application, an example of a MAP abelian group $G$ is given such that every closed, metrizable subgroup $N$ of $b G$ with $N \cap G=\{0\}$ preserves compactness but $G$ does not strongly respects compactness (see Definition 5.3 below). Thereby, we respond to Questions 4.1 and 4.3 in [6].

Date: January 11, 2019.

2010 Mathematics Subject Classification. Primary: 22B05; Secondary: 54H11.

Key words and phrases. abelian group, weak topologies, Weil completion, Bohr compactification, locally compact group, maximally almost periodic group, hemicompactness.

The first listed author acknowledges partially support by the Spanish Ministerio de Economía y Competitividad, grant MTM2016-77143-P (AEI/FEDER, EU), and the Universitat Jaume I, grant P1171B2015-77. 


\section{Preliminaries}

Let $G$ be an abelian group and let $\operatorname{Hom}(G, \mathbb{T})$ be the group of all homomorphisms of $G$ into $\mathbb{T}$. If $X \subseteq G$ and $\Gamma \subseteq \operatorname{Hom}(G, \mathbb{T})$, the symbolism $\left(X, t_{p}(\Gamma)\right)$ (resp. $\left.\left(\Gamma, t_{p}(X)\right)\right)$ denotes the set $X$ (resp. $\Gamma$ ) equipped with the finite-open (or pointwise convergence) topology on the elements of $\Gamma$ (resp. on the elements of $X$ ). A celebrated result by Comfort and Ross [5] establishes that a topological abelian group $(G, \mathrm{w})$ is precompact if and only if there is a subgroup $X \subseteq \operatorname{Hom}(G, \mathbb{T})$ such that w $=t_{p}(X)$. However, in order to keep the notation as simple as possible, we will simply use the symbol "w" to denote a totally bounded (precompact and Hausdorff) group topology on an abelian group $G$ if there is no ambiguity in it. Unless it is explicitly written otherwise, all the topologies considered in this article are assumed to be Hausdorff. In the same manner, if $G$ is an abelian group, the symbol " $\tau$ " stands for a Hausdorff locally compact group topology on $G$. Therefore $\tau^{+}$stands for the Bohr topology on $G$, associated to $\tau$ (we will simply write $G^{+}$when there is no ambiguity about the topology on $G$ ). For example, $\mathbb{R}^{+}$stands for the usual real numbers group equipped with its Bohr topology. If $\tau$ is the discrete topology, we write $\tau^{\#}$ instead of $\tau^{+}$; we could also write $G^{\#}$ to mean the group $G$ equipped with its largest totally bounded group topology. For example, $\mathbb{Z}^{\#}$ stands for the integers with its Bohr topology. We identify the torus $\mathbb{T}$ with the group $[0,1) \subset \mathbb{R}$ equipped with the operation $+\bmod 1$. If $(G, t)$ is a topological abelian group and $H$ is a subgroup of $G$, then $\left(H, t_{\uparrow_{H}}\right)$ stands for the group $H$ equipped with the topology inherited from $(G, t)$. Writing $\left(H, t_{\uparrow_{H}}\right)<(G, t)$ means that $\left(H, t_{\uparrow_{H}}\right)$ is a closed subgroup of $(G, t)$. If so, $(G / H, t / H)$ stands for the natural quotient group.

For each topological abelian group $(G, t)$, the symbol $(G, t)^{\wedge}$ stands for the set of $t$-continuous homomorphisms from $G$ to $\mathbb{T}$. $(G, t)^{\wedge}$ is an abelian group that is called the group of characters of $(G, t)$. Again, in order to simplify the notation, we will denote by $\left((G, t)^{\uparrow}, t_{p}\right)$ the group $\left((G, t)^{\wedge}, t_{p}(G)\right)$. In like manner, the symbolism $\left((G, t)^{\wedge}, t_{k}(G)\right)$, or just $\left((G, t)^{\wedge}, t_{k}\right)$ for short, will denote the group $(G, t)^{\wedge}$ equipped with the compact-open topology on the compact subsets of $(G, t)$. The group $\left((G, t)^{\wedge}, t_{k}\right)$ is known as the dual group of $(G, t)$. There is a canonical homomorphism of $(G, t)$ into its bidual group

$$
\left.\Omega:(G, t) \longrightarrow\left((G, t)^{\uparrow} t_{k}\right)^{\uparrow}, t_{k}\right)
$$

given by

$$
\Omega(g)(\chi)=\chi(g)
$$

for $\chi \in(G, t)^{\wedge}$ and $g \in G$. The map $\Omega$ is called evaluation map of $(G, t)$. It is not hard to see that

- $\Omega$ is injective iff for all $e_{G} \neq x \in G$ there is a character $\chi \in \widehat{G}$ such that $\chi(x) \neq 1$;

- $\Omega$ is continuous when $(G, t)$ is locally compact.

If $H$ is a subgroup of $G$, the annihilator of $H$ in $(G, t)^{\wedge}$, denoted by $\mathbb{A}\left((G, t)^{\wedge}, H\right)$ is the subgroup of $(G, t)^{\wedge}$ consisting of those characters $\varphi$ from $G$ to $\mathbb{T}$, such that $\varphi[H]=\{0\}$. If $X$ is a subgroup of $\operatorname{Hom}(G, \mathbb{T})$, we say that $X$ separates the points of $G$ if whenever $g \in G$ and $g \neq 0_{G}$, the identity of $G$, then there is $\varphi \in X$ such that $\varphi(g) \neq 0$.

Theorem 2.1. Let $G$ be an abelian group. The following assertions hold true:

(1) If $(G, \tau)$ is locally compact $\left((G, \tau)^{\wedge}, t_{k}\right)$ is locally compact.

(2) if $(G, \tau)$ is discrete (resp., compact) then $\left((G, \tau)^{\uparrow}, t_{k}\right)$ is compact (resp. discrete).

(3) If $(G, \tau)$ is locally compact then $\Omega$ is a topological isomorphism of $(G, \tau)$ onto its bidual group.

(4) The group $\left(\operatorname{Hom}(G, \mathbb{T}), t_{p}(G)\right)$ is compact.

(5) If $X \subseteq \operatorname{Hom}(G, \mathbb{T})$, then $\left(X, t_{p}(G)\right)$ is precompact.

(6) If $(G, \mathrm{w})$ is precompact and $X=(G, \mathrm{w})^{\wedge}$, then $(G, \mathrm{w})=\left(G, t_{p}(X)\right)$.

(7) If $X$ is a subgroup of $\operatorname{Hom}(G, \mathbb{T})$, then $X$ separates the points of $G$ if and only if the group $\left(G, t_{p}(X)\right)$ is Hausdorff (resp. if and only if $X$ is dense in $\left.\left(\operatorname{Hom}(G, \mathbb{T}), t_{p}(G)\right)\right)$.

(8) The evaluation map $\Omega:(G, \mathrm{w}) \longrightarrow\left(\left((G, w)^{\uparrow}, t_{p}\right)^{\uparrow}, t_{p}\right)$ is a topological isomorphism onto.

(9) If $(G, \tau)$ is locally compact, then $K$ is a compact subspace of $(G, \tau)$ if and only if $K$ is a compact subspace of $\left(G, \tau^{+}\right)$.

(10) If $\tau$ is locally compact and $\left(H, \tau_{\uparrow H}\right)<(G, \tau)$, then the Bohr topology of $\left(H, \tau_{\uparrow H}\right)$ as a locally compactgroup is the same as the topology that it inherits as a subgroup of $\left(G, \tau^{+}\right)$. 
Proof: 1 and 2 are the celebrated Pontryagin-van Kampen theorem. 3 follows from 1 taking $\tau$ discrete. 4 and 5 follow from Comfort and Ross [5]. 6 is [14] (26.16). 7 is Raczkowski and Trigos-Arrieta [18]. 8 is Glicksberg's Theorem [11]. 9 is done in [20].

\section{NeCESSARY CONDITIONS.}

In the sequel, the symbolism $(G, \mathrm{w})$ will denote a totally bounded abelian group.

Theorem 3.1. If $(G, \mathrm{w})$ is such that $\left((G, \mathrm{w})^{\wedge}, t_{k}\right)$ is locally compact, then $(\bar{G}, \tau):=\left(\left((G, \mathrm{w})^{\wedge}, t_{k}\right)^{\wedge}, t_{k}\right)$ satisfies that $(G, \mathrm{w})$ is contained in $\left(\bar{G}, \tau^{+}\right)$densely.

Proof: The identity $I:\left((G, \mathrm{w})^{\wedge}, t_{k}\right) \longrightarrow\left((G, \mathrm{w})^{\wedge}, t_{p}\right)$, is clearly continuous and onto. Hence, the adjoint

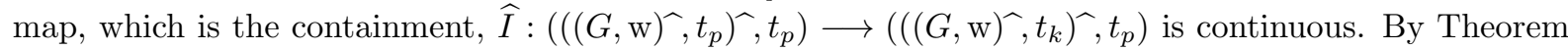
2.1(8), $\left(\left((G, \mathrm{w})^{\uparrow}, t_{p}\right)^{\wedge}, t_{p}\right)=(G, \mathrm{w})$. By Theorem 2.1(1), the group $(\bar{G}, \tau)$ is locally compact. Hence, $\left(\bar{G}, \tau^{+}\right)=\left(\left((G, \mathrm{w})^{\widehat{ }}, t_{k}\right)^{\uparrow}, t_{p}\right)$. That the containment is dense, follows from Theorem 2.1(7).

The following is one of the principal subjects of this paper.

Definition 3.2. Let $\mathcal{B}:=\left\{(G, \mathrm{w}): \exists \tau\left[\mathrm{w}=\tau^{+}\right]\right\}$. Members of the class $\mathcal{B}$ will be called Bohr groups.

Notice that $(G, \mathrm{w}) \in \mathcal{B} \Longrightarrow(G, \mathrm{w})^{\wedge}=\left(G, \tau^{+}\right)^{\wedge}=(G, \tau)^{\uparrow}$, for some locally compact topological abelian group topology $\tau$.

Lemma 3.3. If $(G, \mathrm{w}) \in \mathcal{B}$, then $\left((G, \mathrm{w})^{\wedge}, t_{k}\right)$ is locally compact.

Proof: Suppose that $\tau$ is locally compact with $\tau^{+}=\mathrm{w}$. By Theorem 2.1.8, $\left((G, \mathrm{w})^{\wedge}, t_{k}\right)=\left((G, \tau)^{\wedge}, t_{k}\right)$, which is locally compact by Theorem 2.1.1.

Theorem 3.4. Let $H$ be a closed subgroup of $(G, \mathrm{w})$. If $\left(H, \mathrm{w}_{\uparrow H}\right) \in \mathcal{B}$, and $(G / H, \mathrm{w} / H) \in \mathcal{B}$, then $(G, \mathrm{w}) \in \mathcal{B}$.

Proof: By Lemma 3.3, both $\left((G / H, \mathrm{w} / H)^{\widehat{ }}, t_{k}\right)$ and $\left(\left(H, \mathrm{w}_{\uparrow H}\right)^{\uparrow}, t_{k}\right)$ are locally compact. Let $X:=$ $\left((G, \mathrm{w})^{\wedge}, t_{k}\right)$. We have that $(G / H, \mathrm{w} / H)^{\uparrow}=\mathbb{A}(X, H)[14](23.25 \& 23.30)$ and $\left(H, \mathrm{w}_{\uparrow H}\right)^{\wedge}=X / \mathbb{A}(X, H)$ [14] (24.11 \& 23.30). By [14] (5.25), we have that $X$ is locally compact, hence $(\bar{G}, \tau):=\left(X^{\wedge}, t_{k}\right)$ is locally compact as well. It follows that $G \subseteq(\bar{G}, \tau)$ densely, since both $G$ and $\bar{G}$ separate the points of $X$. We claim that $H$ as a subgroup of $(\bar{G}, \tau)$ is closed. For, $(H, \mathrm{w} \uparrow H) \in \mathcal{B}$ implies $\exists \tau_{H}$ locally compact such that $\left(H, \tau_{H}^{+}\right)=\left(H, \mathrm{w}_{\uparrow H}\right)$. The latter implies that $\left(H, \tau_{H}\right)^{\uparrow}=\left(H, \tau_{H}^{+}\right)^{\uparrow}=\left(H, \mathrm{w}_{\uparrow H}\right)^{\uparrow}=X / \mathbb{A}(X, H)$. Therefore, $\left(H, \tau_{H}\right)=(X / \mathbb{A}(X, H))^{\wedge} \leq(\bar{G}, \tau)$ which proves the claim. Similarly, we claim that $G / H$ is locally compact as a subgroup of $(\bar{G} / H, \tau / H)$. For, $(G / H, \mathrm{w} / H) \in \mathcal{B}$ implies $\exists \tau_{G / H}$ locally compact such that

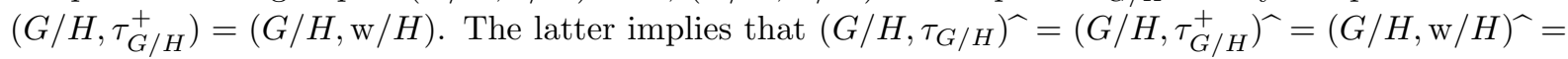
$\mathbb{A}(X, H)$. Therefore, $\left(G / H, \tau_{G / H}\right)=\mathbb{A}(X, H)^{\wedge}=\bar{G} / H$; proving the second claim. Since $H$ and $G / H$ are locally compact as a subgroup and quotient of $G \subseteq(\bar{G}, \tau)$ respectively, it follows, by [14] (5.25), that $G$ is locally compact as a subgroup of $(\bar{G}, \tau)$. Because $G$ is dense in $\bar{G}$, it follows that $G=\bar{G}[14](5.11)$, hence, $\left(G, \tau^{+}\right)=(G, \mathrm{w})$, as required.

Theorem 3.5. Assume all compact subsets of $(G, \mathrm{w})$ are finite. Then TFAE:

(1) $(G, \mathrm{w}) \in \mathcal{B}$.

(2) $\mathrm{w}$ is the largest totally bounded group topology on $G$.

(3) $\left((G, \mathrm{w})^{\wedge}, t_{p}\right)$ is compact.

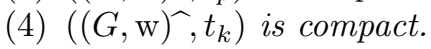

(5) $\left((G, \mathrm{w})^{\wedge}, t_{p}\right)$ is locally compact.

(6) $\left((G, \mathrm{w})^{\wedge}, t_{k}\right)$ is locally compact.

(7) Every homomorphism $f:(G, \mathrm{w}) \longrightarrow \mathbb{T}$ is continuous.

Proof: The hypothesis on the compact subsets of $(G, \mathrm{w})$ implies that $\left((G, \mathrm{w})^{\uparrow}, t_{p}\right)=\left((G, \mathrm{w})^{\uparrow}, t_{k}\right)$. Therefore $3 \Longleftrightarrow 4$ and $5 \Longleftrightarrow 6.2 \Longrightarrow 1$ is obvious, Lemma 3.3 yields $1 \Longrightarrow 6$. Because every group of characters equipped with the finite-open topology is totally bounded, $5 \Longrightarrow 3$. Obviously, $2 \Longleftrightarrow 7$. $3 \Longrightarrow 7$ can be seen by using (3), (4) and (6) of Theorem 2.1.

By [14] (5.14), every locally compact abelian group $(G, \tau)$ contains an open compactly generated subgroup $\left(H, \tau_{\uparrow H}\right)$. Notice then that $(G / H, \tau / H)$ is discrete. By properties of the Bohr topology, we have 
then that $\left(G, \tau^{+}\right)$contains a compactly generated subgroup $\left(H, \tau_{\uparrow H}^{+}\right)$such that $\left(G / H, \tau^{+} / H\right)=(G / H)^{\#}$. This proves the sufficiency of the following:

Lemma 3.6. $(G, \mathrm{w}) \in \mathcal{B}$ if and only if there is a compactly generated subgroup $\left(H, \mathrm{w}_{\uparrow H}\right)$ of $(G, \mathrm{w})$, such that $\left(H, \mathrm{w}_{\uparrow H}\right) \in \mathcal{B}$ and $(G / H, \mathrm{w} / H)=(G / H)^{\#}$.

Proof: For the necessity, use Theorem 3.4.

Corollary 3.7. If for every compactly generated subgroup $\left(H, \mathrm{w}_{\uparrow H}\right)$ of $(G, \mathrm{w})$ we have that $\left(H, \mathrm{w}_{\uparrow H}\right) \notin \mathcal{B}$ or $(G / H, \mathrm{w} / H) \neq(G / H)^{\#}$, then $(G, \mathrm{w}) \notin \mathcal{B}$.

By [14] (9.8) a compactly generated locally compact abelian group must have the form $\mathbb{Z}^{m} \times \mathbb{R}^{n} \times K$ where $m, n$ are non-negative integers and $K$ is a compact abelian group. Because of Theorem 2.1.8 and properties of the Bohr topology, a compactly generated precompact group $(G, \mathrm{w}) \in \mathcal{B}$ if and only if it has the form $\left(\mathbb{Z}^{\#}\right)^{m} \times\left(\mathbb{R}^{+}\right)^{n} \times K$ where $m, n$ are non-negative integers and $K$ is a compact abelian group, and by duality properties, if $(G, \mathrm{w}) \in \mathcal{B}$, then $\left((G, \mathrm{w})^{\wedge}, t_{k}\right)$ has the form $\mathbb{T}^{m} \times \mathbb{R}^{n} \times D$, where $m, n$ are non-negative integers and $D$ is a discrete abelian group.

Theorem 3.8. Assume that $(G, \mathrm{w})$ is compactly generated. Then $1 \Longleftrightarrow 2 \Longrightarrow 3$ :

(1) $(G, \mathrm{w}) \in \mathcal{B}$,

(2) $(G, \mathrm{w})=\left(\mathbb{Z}^{\#}\right)^{m} \times\left(\mathbb{R}^{+}\right)^{n} \times K$, where $m, n$ are non-negative integers and $K$ is a compact abelian group

(3) $\left((G, \mathrm{w})^{\wedge}, t_{k}\right)=\mathbb{T}^{m} \times \mathbb{R}^{n} \times D$, where $m, n$ are non-negative integers and $D$ is a discrete abelian group.

Notice that (3) does not imply (2) above: Setting $(G, \mathrm{w}):=\mathbb{Q}^{+}$, one has $\left((G, \mathrm{w})^{\wedge}, t_{k}\right)=\mathbb{R}$.

Theorem 3.9. Assume that $(G, \mathrm{w})$ is compactly generated and 0-dimensional. Then $(G, \mathrm{w}) \in \mathcal{B}$ if and only if the subgroup $B$ of $(G, \mathrm{w})$ of all compact elements of $(G, \mathrm{w})[14,(9.10)]$ is compact, and $(G / B, \mathrm{w})=(G / B)^{\#}$.

Proof: $(\Longrightarrow)$ Assume that $(G, \mathrm{w})=\left(G, \tau^{+}\right)$. By [14] (9.8) there are $m, n \in \omega$ and a compact group $K$ such that $(G, \tau)=\mathbb{R}^{m} \times \mathbb{Z}^{n} \times K$. By hypothesis, $m=0$ and $K$ is 0 -dimensional. It follows that $(G, \mathrm{w})=\left(G, \tau^{+}\right)=\left(\mathbb{Z}^{\#}\right)^{n} \times K, B=K$, and $(G / B, \mathrm{w})=\left(\mathbb{Z}^{\#}\right)^{n}$, as required. For $(\Longleftarrow)$, apply Theorem 3.4 .

Lemma 3.10. Suppose the groups $A, B$ and $G$ satisfy $(G, \mathrm{w})=\left(A, \mathrm{w}_{1}\right) \times\left(B, \mathrm{w}_{2}\right)$. Then $(G, \mathrm{w}) \in \mathcal{B}$ if and only if both $\left(A, \mathrm{w}_{1}\right) \in \mathcal{B}$ and $\left(B, \mathrm{w}_{2}\right) \in \mathcal{B}$.

Proof: By the main hypothesis, $\left((G, \mathrm{w})^{\wedge}, t_{k}\right)=\left(\left(A, \mathrm{w}_{1}\right)^{\wedge}, t_{k}\right) \times\left(\left(B, \mathrm{w}_{2}\right)^{\Upsilon}, t_{k}\right)$. If $(G, \mathrm{w}) \in \mathcal{B}$, say $(G, \mathrm{w})=$ $\left(G, \tau^{+}\right)$, then, by Theorem 2.1.2, $(G, \tau)=\left(\left((G, \mathrm{w})^{\wedge}, t_{k}\right)^{\wedge}, t_{k}\right)=\left(\left(\left(A, \mathrm{w}_{1}\right)^{\wedge}, t_{k}\right)^{\wedge}, t_{k}\right) \times\left(\left(\left(B, \mathrm{w}_{2}\right)^{\uparrow}, t_{k}\right)^{\uparrow}, t_{k}\right)$. We write $\left(\bar{A}, \tau_{1}\right):=\left(\left(\left(A, \mathrm{w}_{1}\right)^{\Upsilon}, t_{k}\right)^{\Upsilon}, t_{k}\right)$, and $\left(\bar{B}, \tau_{2}\right):=\left(\left(\left(B, \mathrm{w}_{2}\right)^{\Upsilon}, t_{k}\right)^{\Upsilon}, t_{k}\right)$. Hence $(G, \tau)=\left(\bar{A}, \tau_{1}\right) \times$ $\left(\bar{B}, \tau_{2}\right)$, and by Theorem 3.1, $\left(A, \tau_{1 \mid A}\right)$ is a dense subgroup of $\left(\bar{A}, \tau_{1}\right)$, and $\left(B, \tau_{2 \nmid B}\right)$ is a dense subgroup of $\left(\bar{B}, \tau_{2}\right)$. But since $\left(A, \mathrm{w}_{1}\right) \times\{0\} \leq(G, \mathrm{w})$, it follows that $\left(A, \tau_{1}\right) \times\{0\} \leq(G, \tau)$. Similarly, $\{0\} \times\left(B, \tau_{2}\right) \leq$ $(G, \tau)$. Thus both $\left(A, \tau_{1}\right)$ and $\left(B, \tau_{2}\right)$ are locally compact, hence $\left(A, \tau_{1 \uparrow A}\right)=\left(\bar{A}, \tau_{1}\right)$ and $\left(B, \tau_{2 \uparrow B}\right)=$ $\left(\bar{B}, \tau_{2}\right)$. By properties of the Bohr topology, $\left(A, \mathrm{w}_{1}\right) \times\left(B, \mathrm{w}_{2}\right)=(G, \mathrm{w})=\left(G, \tau^{+}\right)=\left(A, \tau_{1}^{+}\right) \times\left(B, \tau_{2}^{+}\right)$. This obviously implies that $\left(A, \mathrm{w}_{1}\right)=\left(A, \tau_{1}^{+}\right)$and $\left(B, \mathrm{w}_{2}\right)=\left(B, \tau_{2}^{+}\right)$. The converse is obvious.

Theorem 3.11. The topological group $(G, \mathrm{w}) \in \mathcal{B}$ if and only if there are groups $A, B$ and $C$ such that (a) $G=A \times B$, (b) $\left(A \times\{0\}, \mathrm{w}_{\uparrow(A \times\{0\})}\right)=\left(\mathbb{R}^{+}\right)^{n}$ for some $n \in \omega$, and (c) $\left(\{0\} \times C, \mathrm{w}_{\uparrow(\{0\} \times C)}\right)$ is a compact subgroup of $\left(\{0\} \times B, \mathrm{w}_{\uparrow(\{0\} \times B)}\right)$ such that $(B / C, \mathrm{w} / C)=(B / C)^{\#}$.

Proof: $(\Longrightarrow)$ Assume that $(G, \mathrm{w})=\left(G, \tau^{+}\right)$. By [14] (24.30) there are $n \in \omega$, a locally compact group $G_{0}$ and a compact group $K$ such that $(G, \tau)=\mathbb{R}^{n} \times G_{0}$, and $\left(G_{0} / K, \tau / K\right)$ is discrete. Let $A, B$ and $C$ be the underlying groups of $\mathbb{R}^{n}, G_{0}$, and $K$, respectively. By properties of the Bohr topology, we have that (a), (b) and (c) hold. For $(\Longleftarrow)$, apply Theorem 3.4 to see that $\left(\{0\} \times B, \mathrm{w}_{\uparrow(\{0\} \times B)}\right) \in \mathcal{B}$. That $(G, \mathrm{w}) \in \mathcal{B}$ follows after an application of Lemma 3.10.

Theorem 3.12. Consider the topological abelian group $(G, \mathrm{w})$. Suppose that $F$ is its connected component. Then $(G, \mathrm{w}) \in \mathcal{B}$ if and only if both $\left(F, \mathrm{w}_{\uparrow F}\right) \in \mathcal{B}$, and $(G / F, \mathrm{w} / F) \in \mathcal{B}$. 
Proof: $(\Longrightarrow)$ Assume that $(G, \mathrm{w})=\left(G, \tau^{+}\right)$. By [14] (24.30) there are $n \in \omega$, a locally compact group $G_{0}$ and a compact group $K$ such that $(G, \tau)=\mathbb{R}^{n} \times G_{0}$, and $\left(G_{0} / K, \tau / K\right)$ is discrete. Let $C$ be the connected component of $G_{0}$. Clearly, $\left(C, \tau^{+}\right) \leq\left(G_{0}, \tau^{+}\right)=\left(G_{0}, \mathrm{w}\right)$, with $\left(C, \tau^{+}\right)=(C, \mathrm{w})$ connected. Since $\left(G_{0} / C, \tau\right)$ is 0-dimensional, it follows, by properties of the Bohr topology [12], that $\left(G_{0} / C, \tau^{+}\right)$is $0-$ dimensional. If $F:=\left(\mathbb{R}^{+}\right)^{n} \times C$, it follows that $(F, \mathrm{w}) \in \mathcal{B}$ is the connected component of $(G, \mathrm{w})=\left(G, \tau^{+}\right)$. Of course $(G / F, \mathrm{w})=\left(G / F, \tau^{+}\right) \in \mathcal{B}$. For $(\Longleftarrow)$, apply Theorem 3.4.

Corollary 3.13. The following are equivalent for a topological abelian group $(G, \mathrm{w})$.

(1) $(G, \mathrm{w}) \in \mathcal{B}$.

(2) There are groups $A, B$ and $C$ such that (a) $G=A \times B$, (b) $\left(A \times\{0\}, \mathrm{w}_{\uparrow(A \times\{0\})}\right)=\left(\mathbb{R}^{+}\right)^{n}$ for some $n \in \omega$, and $(c)\left(\{0\} \times C, \mathrm{w}_{\uparrow(\{0\} \times C)}\right)$ is a compact subgroup of $\left(\{0\} \times B, \mathrm{w}_{\uparrow(\{0\} \times B)}\right)$ such that $(B / C, \mathrm{w} / C)=(B / C)^{\#}$.

(3) If $F$ is the connected component of $(G, \mathrm{w}) \in \mathcal{B}$ then $\left(F, \mathrm{w}_{\uparrow F}\right) \in \mathcal{B}$, and $(G / F, \mathrm{w} / F) \in \mathcal{B}$.

(4) There is a compactly generated subgroup $\left(H, \mathrm{w}_{\uparrow H}\right)$ of $(G, \mathrm{w})$, such that $\left(H, \mathrm{w}_{\uparrow H}\right) \in \mathcal{B}$ and $(G / H, \mathrm{w} / H)=$ $(G / H)^{\#}$.

Corollary 3.14. If $(G, \mathrm{w})$ is a metrizable Hausdorff group in $\mathcal{B}$, then $(G, \mathrm{w})$ is compact.

\section{Some topologies CANONicAlly ASSOCIATEd to topological Groups}

Following the terminology in [6], for a (Hausdorff) space $X=(X, t)$ we denote by $k X$, or $(X, k t)$, the set $X$ with the topology $k t$ defined as follows: A subset $U$ of $X$ is $k t$-open if and only if $U \cap K$ is (relatively) $t$-open in $K$ for every $t$-compact subset $K$ of $X$. Then $k X$ is a $k$-space (that is, $k X=k k X$ ), $k t$ is the smallest $k$-space topology on $X$ containing $t$. Furthermore $k t$ is the unique $k$-space topology on $X$ larger than $t$ such that each $t$-compact subset of $X$ is also $k t$-compact. In like manner, we say that a map $f$ defined on $X$ is $k$-continuous when $f$ is continuous on each compact subset of $X$.

Given a topological abelian group $(G, t)$ with dual $X:=\widehat{(G, t)}$, for any subset $A$ of $G$, we define $A^{0}:=\left\{\chi \in X:|\chi(g)| \leq \frac{1}{4} \forall g \in A\right\}$. Assuming that we are considering the dual pair $(G, X)$, for any subset $L$ of $X$, we define $L^{0}:=\left\{g \in G:|\chi(g)| \leq \frac{1}{4} \forall \chi \in L\right\}$. This set operator behaves in many aspects like the polar operator in vector spaces. For instance, it is easily checked that $A^{000}=A^{0}$ for any $A \subset G$. Given an arbitrary subset $A$ in $G$, we define the quasi convex hull of $A$, denoted $c o(A)$, as the set $A^{00}$. A set $A$ is said to be quasi convex when it coincides with its quasi convex hull. These definitions also apply to subsets $L$ of $X$. The topological group $(G, t)$ is said to be locally quasi convex when there is a neighborhood base of the identity consisting of quasi convex sets.

Let $(G, t)$ be a MAP topological abelian group. In the sequel we are going to look at the following natural group topologies canonically attached to $(G, t)$.

Definition 4.1. (1) The Bohr topology. Denoted by $t^{+}$, it is the weak topology generated by the $t$-continuous homomorphisms from $G$ into $\mathbb{T}$. It easily seen that the canonical map $b:(G, t) \mapsto$ $\left(G, t^{+}\right)$is an epireflective functor from the category of topological groups into the subcategory of Hausdorff precompact groups.

(2) The locally quasi convex topology. Denoted by $q[t]$, it is the finest locally quasi convex topology that is contained in $t$. Again, it easily seen that the canonical map $q:(G, t) \mapsto(G, q[t])$ is an epireflective functor from the category of topological groups into the subcategory of locally quasi convex groups.

(3) The $g$-sequential topology. Denoted by $s_{g}[t]$, it is the finest group topology coarser than the sequential modification of $t$, i.e., the largest topology on $G$ with the same $t$-convergent sequences. When $t=s_{g}[t]$, it is said that $(G, t)$ is a $g$-sequential group. The canonical map $g:(G, t) \mapsto\left(G, s_{g}[t]\right)$ defines a coreflective functor from the category of topological groups into the subcategory of $g$-groups.

(4) The $k_{g}$-topology. Denoted by $k_{g}[t]$, it was originally defined by Noble [17] as the supreme of all group topologies on $G$ that lie between $t$ and the $k$-topology $k t$ attached to $t$. When $t=k_{g}[t]$, it is said that $(G, t)$ is a $k_{g}$-group. As in $(3)$, the canonical map $k_{g}:(G, t) \longrightarrow\left(G, k_{g}[t]\right)$ is a coreflective functor from the category of topological groups into the subcategory of $k_{g}$-groups. 
First, we explore the relationship among these topologies. The topologies (3) and (4) have very similar properties and we will only present the proofs for the $g$-sequential topology since basically the same proofs work for the $k_{g}$-topology.

Lemma 4.2. Let $(G, \mathrm{w})$ be a totally bounded group. Then $(G, \mathrm{w})$ is the Bohr reflection of a g-sequential group if and only if $\mathrm{w}$ is the finest among all totally bounded topologies on $G$ that share the same collection of convergent sequences. If either condition holds, then $\left(G, s_{g}[\mathrm{w}]\right)$ has the same dual as $(G, \mathrm{w})$.

Proof: Assume that $\mathrm{w}=t^{+}$for some $g$-sequential topology $t$ on $G$. Let $\rho$ be another totally bounded topology on $G$ such that w and $\rho$ have the same family of convergent sequences. Then the identity mapping $1_{G}:\left(G, s_{g}[\mathrm{w}]\right) \longrightarrow(G, \rho)$ is sequentially continuous and, therefore, continuous as well. Now, since the map $g$ defines a correflective functor and $(G, t)$ is a $g$-sequential group, from $t \geq \mathrm{w}$, it follows that $\tau \geq s_{g}[\mathrm{w}]$, hence $1_{G}:(G, \tau) \longrightarrow(G, \rho)$ is continuous. Being $\rho$ totally bounded, this implies that $1_{G}:\left(G, t^{+}\right) \longrightarrow(G, \rho)$ is continuous. Since $t^{+}=\mathrm{w}$, it follows that $\mathrm{w} \geq \rho$.

Conversely, suppose that w is the finest totally bounded topology on $G$ with the same family of convergent sequences. First, we will see that $\left(G, s_{g}[\mathrm{w}]\right)$ has the same dual as $(G, \mathrm{w})$. Trivially, every w-continuous character is automatically $s_{g}[\mathrm{w}]$-continuous. We claim that if $\chi$ were a $s_{g}[\mathrm{w}]$-continuous character that is not $\mathrm{w}$-continuous, then $\mathrm{w} \vee t_{\chi}$, the supreme topology generated by $\mathrm{w}$ and the initial topology generated by $\chi$, would be a totally bounded topology with the same convergent sequences as $\mathrm{w}$.

Indeed, let $\left(x_{i}\right)$ be a sequence in $G$ w-converging to some point $x_{0} \in G$. Since every w-convergent sequence is $s_{g}[\mathrm{w}]$-convergent, it follows that $\left(x_{i}\right) s_{g}[\mathrm{w}]$-converges to $x_{0}$. As a consequence $\left(\chi\left(x_{i}\right)\right)$ converges to $\chi\left(x_{0}\right)$, and therefore, $\left(x_{i}\right)$ converges to $x_{0}$ in $\mathrm{w} \vee t_{\chi}$. Thus $\mathrm{w}=\mathrm{w} \vee t_{\chi}$, which means that $\chi$ is wcontinuous by Theorem 2.1.5. We have therefore verified that $\mathrm{w}$ is the Bohr topology associated to $s_{g}[\mathrm{w}]$.

Lemma 4.3. Let $(G, \mathrm{w})$ be a totally bounded group. Then $(G, \mathrm{w})$ is the Bohr reflection of a $k_{q}$-group if and only if $\mathrm{w}$ is the finest among all totally bounded topologies on $G$ that share the same collection of compact subsets. If either condition holds, then $\left(G, k_{g}[\mathrm{w}]\right)$ has the same dual as $(G, \mathrm{w})$.

Corollary 4.4. A countable totally bounded group $(G, \mathrm{w})$ is in $\mathcal{B}$ if and only if every character of $G$ is $\mathrm{w}$-continuous. In this case, the groups $\left(G, s_{g}[\mathrm{w}]\right)$ and $\left(G, k_{g}[\mathrm{w}]\right)$ are discrete.

Proof: $(\Longrightarrow)$ follows from Corollary 3.13, while $(\Longleftarrow)$ follows from Theorem 2.1.5 and Corollary 3.13.2. That $s_{g}[\mathrm{w}]$ and $k_{g}[\mathrm{w}]$ are the discrete topology follows from Theorem 3.5.

Corollary 4.5. Let $(G, \mathrm{w})$ be a totally bounded group that is the Bohr reflection of a g-sequential (resp. $\left.k_{g}\right)$ group $(G, \tau)$. Then $\mathrm{w}=s_{g}[\mathrm{w}]^{+}\left(\right.$resp. $\left.\mathrm{w}=k_{g}[\mathrm{w}]^{+}\right)$.

Proof: It suffices to notice that $\left(G, s_{g}[\mathrm{w}]\right)\left(\left(G, k_{g}[\mathrm{w}]\right)\right.$, resp.) has the same dual as $(G, \mathrm{w})$.

Lemma 4.6. Let $(G, t)$ be a topological abelian group and let $G^{\prime}:=(G, t)^{\wedge}$ denote its dual group. Then the following are equivalent:

(1) Every sequentially continuous character on $(G, t)$ is continuous;

(2) $\left(G, s_{g}[t]\right)^{\wedge}=G^{\prime}$;

(3) $\left(G, s_{g}[t]^{+}\right)=\left(G, t^{+}\right)$;

(4) There exists a g-sequential topology $\tilde{t}$ on $G$ such that $t \subseteq \tilde{t}$ and $t^{+}=\tilde{t}^{+}$.

Lemma 4.7. Let $(G, t)$ be a topological abelian group and let $G^{\prime}:=(G, t)^{\wedge}$ denote its dual group. Then the following are equivalent:

(1) Every $k$-continuous character on $(G, t)$ is continuous;

(2) $\left(G, k_{g}[t]\right)^{\wedge}=G^{\prime}$;

(3) $\left(G, k_{g}[t]^{+}\right)=\left(G, t^{+}\right)$;

(4) There exists a $k_{g}$-topology $\tau$ on $G$ such that $t \subseteq \tau$ and $t^{+}=\tau^{+}$.

Corollary 4.8. The group $(G, t)$ respects convergent sequences if and only if $t \subseteq s_{g}\left[t^{+}\right]$.

Corollary 4.9. The group $(G, t)$ respects compact subsets if and only if $t \subseteq k_{g}\left[t^{+}\right]$.

In order to characterize Bohr groups we need two basic notions. The first one is well known and the later was introduced in [13]. 
A family $\mathcal{N}$ of subsets of a topological space $X$ is a network at $x \in X$ if for every neighborhood $U$ of $x$ there exists an $M \in \mathcal{N}$ such that $x \in M \subseteq U$. If $\mathcal{N}$ is a network at each point in $X$, we say that $\mathcal{N}$ is a network for $X$.

For any topological group $(G, t)$, we say that $W \subset G$ is a $k$-neighborhood of 0 if for any $t$-compact subset $K \subset G$ containing 0 , there exists a neighborhood $U$ of 0 such that $U \cap K \subset W \cap K$. It is not true in general that if $x \in G$ and $W$ is a $k$-neighborhood of $x$ in $(G, t)$ then $W$ is a neighborhood of $x$ in $\left.\left(G, k_{g}[t]\right)\right)$. However, when $W$ is a quasi convex set the assertion above holds true (see Proposition 1 in $[13])$.

A topological space is said to be hemicompact if in the family of all the compact subspaces of $X$ ordered by $\subseteq$ there is a countable cofinal subfamily. The concept was introduced by Arens in [1]. Hemicompact spaces are of course $\sigma$-compact but $\mathbb{Q}$ shows that the containment is proper. We now look at groups that are hemicompact.

Theorem 4.10. Let $(G, \mathrm{w})$ be a hemicompact totally bounded group whose cardinality is not Ulammeasurable. Then $(G, \mathrm{w}) \in \mathcal{B}$ if and only if the following properties hold:

(1) Every sequentially continuous character on $(G, \mathrm{w})$ is continuous.

(2) There exists a compact subgroup $K$ of $G$ such that $(G / K, \mathrm{w} / K)$ has a countable network at 0 consisting of $k$-neighborhoods of 0 .

Proof: First, we notice that a wide use of duality techniques are essential for the proof. Assume that $(G, \mathrm{w}) \in \mathcal{B}$ and let $\tau$ be a locally compact topology on $G$ such that $\tau^{+}=\mathrm{w}$. Then $(G, \tau)$ satisfies the two assertions above. Indeed, that $(G, \tau)$ satisfies (1) is due to results of Varopoulos [21] and Reid [19]. Also, the celebrated Kakutani-Kodaira Theorem [3] (3.7) and Theorem 2.1(9) imply that $G$ contains a compact subgroup $K$ such that $(G / K, \tau / K)$ is metric. Therefore $(G / K, \tau / K)$ will be hemicompact and metric. Now, every LCA group is a locally quasi convex $k_{q}$-group. Therefore, there exists a countable neighborhood base $\mathcal{N}=\left\{U_{n}\right\}$ at the neutral element of $(G / K, \tau / K)$ consisting of $k$-neighborhoods, quasi convex sets. Then $\mathcal{N}$ is a countable network for $G / K$, which proves (2).

Conversely, suppose that (1) and (2) hold. By Theorem 3.4, in order to prove that $(G, \mathrm{w}) \in \mathcal{B}$, it will suffice to verify that $(G / K, \mathrm{w} / K) \in \mathcal{B}$. In other words, there is no loss of generality in assuming that $(G, \mathrm{w})$ has a countable network at $0_{G}$ consisting of $k$-neighborhoods of 0 .

Let us denote by $G^{\prime}:=\left((G, \mathrm{w})^{\wedge}, t_{k}\right)$, the dual topological group of $(G, \mathrm{w})$. Because $(G, \mathrm{w})$ is hemicompact, it follows that $G^{\prime}$ is metric and, by (1), it follows that $G^{\prime}$ is complete metric. Further, by [13, Lemma 5], we have that if $F$ is a $k$-neighborhood of $0_{G}$, then $F^{0}$ is precompact in $G^{\prime}$. Furthermore, being $F^{0}$ closed in $G^{\prime}$, which is complete, it follows that $F^{0}$ is in fact a compact subset of $G^{\prime}$. Since $(G, \mathrm{w})$ has a countable network $\left\{W_{n}\right\}$ at $0_{G}$ consisting of quasi convex subsets, it follows that $G^{\prime}=\bigcup_{n \in \mathbb{N}} W_{n}^{0}$. Therefore $G^{\prime}$ is $\sigma$-compact. Furthermore, since $G^{\prime}$ is complete metric and $\sigma$-compact, by the Baire category theorem, it follows that $G^{\prime}$ is locally compact. In particular, $G^{\prime}$ is hemicompact [1].

Since $(G, \mathrm{w})=\left(G, t_{p}\left(G^{\prime}\right)\right.$ by Theorem 2.1(6), we have the following commuting diagram:

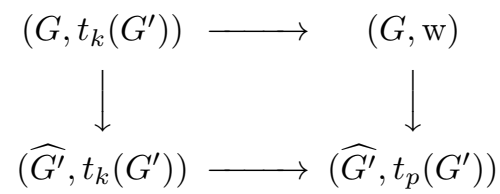

where the evaluation maps given by the vertical arrows are topological embeddings, and the identity maps given by the horizontal arrows are continuous. By Theorem 2.1(9), the totally bounded group $\left(\widehat{G^{\prime}}, t_{p}\left(G^{\prime}\right)\right)$ and the locally compact group $\left(\widehat{G^{\prime}}, t_{k}\left(G^{\prime}\right)\right)$ have the same collection of compact subsets, which implies that $\left(G, t_{k}\left(G^{\prime}\right)\right)$ is itself hemicompact (since $(G, \mathrm{w})$ is hemicompact), and metrizable, as subgroup of $\left(\widehat{G^{\prime}}, t_{k}\left(G^{\prime}\right)\right)$. It follows then, by [1] again, that $\left(G, t_{k}\left(G^{\prime}\right)\right)$ is a locally compact subgroup of $\left(\widehat{G^{\prime}}, t_{k}\left(G^{\prime}\right)\right)$. Therefore, we have proved that $\left.\left(G, t_{k} G^{\prime}\right)\right)$ is locally compact and metric. By Lemma 4.7, its dual group is $G^{\prime}$. By Theorem 2.1(1) it follows that $G=\widehat{G^{\prime}}$ and since $\left(G, t_{k}\left(G^{\prime}\right)\right)^{\wedge}=G^{\prime}=(G, \mathrm{w})^{\wedge}$ we have that $\left(G, t_{k}\left(G^{\prime}\right)^{+}\right)=(G, \mathrm{w})$, hence $(G, \mathrm{w}) \in \mathcal{B}$. This completes the proof.

In case $G$ has Ulam-measurable cardinality, Theorem 4.10 does not hold because there are compact groups that are not $g$-sequential (see [4]). In this case, we must replace sequential continuity by $k$ continuity. 
Theorem 4.11. Let $(G, \mathrm{w})$ be a hemicompact, totally bounded group. Then $(G, \mathrm{w}) \in \mathcal{B}$ if and only if the following properties hold:

(1) Every $k$-continuous character on $(G, \mathrm{w})$ is continuous.

(2) There exists a compact subgroup $K$ of $G$ such that $(G / K, \mathrm{w} / K)$ has a countable network at 0 consisting of $k$-neighborhoods of 0 .

Example 4.12. The hemicompactness condition on Theorems 4.10 and 4.11 cannot be relaxed. If $G=\mathbb{Q}^{+}$, then $G$ satisfies (1) and (2) but $G \notin \mathcal{B}$ since it is not hemicompact [1].

The question of characterizing those totally bounded groups $(G, \mathrm{w})$ such that $(G, k \mathrm{w})$ is locally compact and $(k \mathrm{w})^{+}=\mathrm{w}$ is proposed in [6], and studied further by Galindo [8]. Next, we show how this question is related to the subject matter of this paper.

Lemma 4.13. Let $(G, \mathrm{w})$ be a totally bounded group. Then $(G, \mathrm{w}) \in \mathcal{B}$ if and only if $(G, k \mathrm{w})$ is locally compact and $(k \mathrm{w})^{+}=\mathrm{w}$.

Proof: Sufficiency is obvious. In order to prove necessity, assume that $(G, \mathrm{w}) \in \mathcal{B}$. That is, there is a locally compact topology $\tau$ on $G$ such that $\tau \supseteq \mathrm{w}$ and $\tau^{+}=\mathrm{w}$. Since the topology of every locally compact group is both $g$-sequential and $k$-space (see [21]) and $\tau \supseteq \mathrm{w}$, it follows that $\tau \supseteq s_{g}[\mathrm{w}] \cup k \mathrm{w}$. On the other hand, by Theorem 2.1(9), the groups $(G, \mathrm{w})$ and $(G, \tau)$ have the same collection of compact subsets, which implies that $\tau \subseteq s_{g}[\mathrm{w}] \cap k \mathrm{w}$. In other words, we have that $\tau=s_{g}[\mathrm{w}]=k \mathrm{w}$.

In light of the previous lemma, the next theorem provides an answer to Question 4.3 in [6].

Theorem 4.14. Let $(G, \mathrm{w})$ be a totally bounded group whose cardinality is not Ulam-measurable. Then $(G, \mathrm{w}) \in \mathcal{B}$ if and only if the following properties hold:

(1) Every sequentially continuous character on $(G, \mathrm{w})$ is continuous.

(2) There is a hemicompact subgroup $H$ of $(G, \mathrm{w})$ such that $(G / H, \mathrm{w} / H)=(G / H)^{\#}$

(3) There exists a compact subgroup $K$ of $\left(H, \mathrm{w}_{\uparrow H}\right)$ such that $(H / K, \mathrm{w} / K)$ has a countable network at 0 consisting of $k$-neighborhoods of 0 .

Again, when the group $G$ has Ulam-measurable cardinality, we have the following variant of Theorem 4.14 .

Theorem 4.15. Let $(G, \mathrm{w})$ be a totally bounded group. Then $(G, \mathrm{w}) \in \mathcal{B}$ if and only if the following properties hold:

(1) Every $k$-continuous character on $(G, \mathrm{w})$ is continuous.

(2) There is a hemicompact subgroup $H$ of $(G, \mathrm{w})$ such that $(G / H, \mathrm{w} / H)=(G / H)^{\#}$

(3) There exists a compact subgroup $K$ of $\left(H, \mathrm{w}_{\uparrow H}\right)$ such that $(H / K, \mathrm{w} / K)$ has a countable network at 0 consisting of $k$-neighborhoods of 0 .

We now establish the independence of the three conditions in Theorems 4.14 and 4.15.

Example 4.16. Set $G=(\mathbb{Z}, \mathrm{w})$, where $\mathrm{w}$ is a totally bounded topology on $\mathbb{Z}$ such that $\mathrm{w} \varsubsetneqq t_{p}(\mathbb{T})$, the Bohr topology on $\mathbb{Z}$, but contains no infinite compact subsets (see [6]). Then $G$ satisfies (2) and (3) but fails to satisfy (1). For, (1) does not hold since the only convergent sequences in $G$ are eventually constant; (2) $G$ is obviously hemicompact; and (3) the singleton $\{0\}$ is obviously a countable network at $\{0\}$ consisting of $k$-neighborhoods.

Example 4.17. Let $X$ be an infinite compact metric space, $H_{1}=A(X)^{+}$, where $A(X)$ denotes the free abelian group generated by $X$, and let $H_{2}=\mathbb{R}^{+}$. Set $G=H_{1} \times H_{2}$. Then $G$ satisfies (1) and (2) but fails to satisfy (3). For, a sequentially continuous character of $A(X)$ is obviously continuous on $X$, and thus it will be continuous by the properties of free abelian groups. By [2] (7.4.4) and (7.1.13) $A(X)$ is hemicompact, and by [9] (4.20) $H_{1}$ is hemicompact as well; since $H_{2}$ is hemicompact by Theorem 2.1.8, a simple verification shows that $G$ is also hemicompact. Notice also that if $H_{1}$ satisfied (3), then $\widehat{A(X)}$ would be first countable, hence locally compact, which is absurd [1]. Thus, $G$ does not satisfy (3).

Example 4.18. If $G=\mathbb{Q}^{+}$, then $G$ satisfies (1) and (3) but fails to satisfy (2) [1]. 


\section{Respecting COMPACTNESS}

Let us recall that a group is von Neumann complete if every closed precompact subset of the group is complete.

Lemma 5.1. Let $G$ be a MAP (von Neumann) complete group that respects compactness. If $\left(x_{n}\right)$ is a Cauchy sequence in $G^{+}$, then it converges in $G$.

Proof: If $\left(x_{n}\right)$ is precompact in $G$, then $\overline{\left(x_{n}\right)} G$ is a compact subset in $G$ homeomorphic to $\overline{\left(x_{n}\right)} b G$. As a consequence $\left(x_{n}\right)$ must be convergent in $G$. Therefore, we may assume that $\left(x_{n}\right)$ is not precompact. Furthermore, taking a convenient subsequence if necessary, we may assume that $\left(x_{n}\right)$ is uniformly discrete. That is, there is a neighborhood of the identity, say $U$, such that $x_{n} x_{m}^{-1} \notin U$ for all $n, m$ in $\mathbb{N}$. Now, since $\left(x_{n}\right)$ is Bohr-Cauchy, it follows that $\left(x_{n} x_{n+1}^{-1}\right)$ Bohr-converges to the neutral element. Since $G$ respects compactness, we have that $\left(x_{n} x_{n+1}^{-1}\right)$ converges to the neutral element in $G$, which is a contradiction, completing the proof.

As a consequence of the previous lemma, we obtain:

Lemma 5.2. Let $G$ be a MAP (von Neumann) complete group that respects compactness, and let $N$ be a closed, metrizable subgroup of $b G$. Set $H^{+}=G^{+} \cap N$. Then $H$, the inverse image of $H^{+}$in $G$, is a compact metrizable group isomorphic to $\mathrm{H}^{+}$.

Definition 5.3. We say that a MAP group $G$ strongly respects compactness if whenever $N$ is a closed metrizable subgroup of the Bohr compactification $b G$ of $G$ and $A \subseteq G$, then $A+(N \cap G)$ is compact in $G$ whenever $\phi(A)$ is compact, where $\phi$ is the composition map $G \longrightarrow b G \longrightarrow b G / N$. If $N$ is a closed subgroup of $b G$ such that for any $A \subseteq G, A+(N \cap G)$ is compact in $G$ whenever $\phi(A)$ is compact in $b G / N$, then we say that $N$ preserves compactness.

Obviously, a MAP group that strongly respects compactness also respect compactness, but the converse does not hold [9] (4.10). That locally compact abelian groups strongly respect compactness is the main result in [6], but this has been improved in several directions in [9]. In particular, the next result contributes positively into answering Question 5.1 of [9].

Theorem 5.4. Let $G$ be a MAP abelian Polish group (more generally, a MAP abelian metrizable von Neumann complete group) that respects compactness. Then $G$ strongly respects compactness.

Proof: Let $N$ be closed, metrizable subgroup of $b G$ and assume that $A \subseteq G$ with $\phi(A) \operatorname{compact}$ in $b G / N$. We have to prove that $A+(N \cap G)$ is compact in $G$. Now, since $A+(N \cap G)=(A+N) \cap G$ is closed in $G^{+}$, it is also closed in $G$, which is complete. Therefore, it will suffice to show that $A+(N \cap G)$ is precompact in $G$. Assume otherwise. Then $A+(N \cap G)$ must contain an infinite uniformly discrete sequence $\left(x_{n}\right)=\left(a_{n}+y_{n}\right)$, where $\left(a_{n}\right) \subseteq A$ and $\left(y_{n}\right) \subseteq N \cap G$. Now, $A+N$ is a compact metrizable subspace of $b G$ (in order to see this, use the first part of the proof of Lemma 2.6 in [6]). Therefore, $\left(x_{n}\right)$ must contain a Cauchy subsequence, say $\left(x_{n_{m}}\right)$. By Lemma 5.1, this subsequence converges to a point $p \in G$, which contradicts our assumption about $\left(x_{n}\right)$ being uniformly discrete. Thus $A+(N \cap G)$ is precompact in $G$, which completes the proof.

The following is Question 4.1 in [6]:

Question 5.5. Let $G$ be a MAP group and suppose that every closed, metrizable subgroup $N$ of bG such that $N \cap G=\{0\}$ preserves compactness. Does it follow that $G$ strongly respects compactness?

We will need the following:

Definition 5.6. A Hausdorff topological space $X$ is a $k_{\omega}$-space if there exists an ascending sequence of compact subsets $K_{1} \subseteq K_{2} \subseteq \cdots X$ such that $X=\bigcup_{n \in \omega} K_{n}$ and $U \subseteq X$ is open if and only if $U \cap K_{n}$ is open in $K_{n}$ for each $n<\omega$.

The following result answers Question 5.5 in the negative.

Example 5.7. Take $G_{n}:=\mathbb{T}$ and $H_{n}:=\mathbb{Q} / \mathbb{Z}$, equipped with the topologies inherited from the complex plane and the topology inherited from $\mathbb{R} / \mathbb{Z}$, respectively, for all $n<\omega$, and set $G:=\bigoplus_{n<\omega} G_{n}$, and $H:=\bigoplus_{n<\omega} H_{n}$, with both groups equipped with the box topology. From here on, we identify the groups 
$G_{n}, H_{n}, \bigoplus_{n<N} G_{n}$ and $\bigoplus_{n<N} H_{n}$ with their isomorphic subgroups in $G$ and $H$ respectively. We have the following facts:

(1) $G$ is the countable direct limit of compact groups and, therefore it is a MAP, $k_{\omega}$-group having $H$ as a dense subgroup.

(2) $G$ strongly respects compactness.

(3) For every compact subset $K$ of $G$ there is $n_{0} \in \mathbb{N}$ such that $K \subseteq \bigoplus_{n<n_{0}} G_{n}$.

(4) $b G=b H$.

(5) If $N$ is a compact metrizable subgroup of $b G$ such that $N \cap H=\{0\}$, then $N \cap G=\{0\}$.

(6) If $N$ is a compact metrizable subgroup of $b G$ such that $N \cap H=\{0\}$, then $N$ respects compactness in $H$.

(7) $H$ does not strongly respect compactness.

Proof: (1) is clear. (2) is proved in [7], where it is established that every locally $k_{\omega}$-group strongly respects compactness. (3) is clear since $G$ is equipped with the countable box topology. (4) follows from the density of $H$ in $G$. In order to prove (5), reasoning by contradiction, assume that $N \cap G \neq\{0\}$. Since $G$ strongly respects compactness, it follows that $N \cap G$ is a compact subgroup of $G$ and, by (3), there is $n_{0} \in \mathbb{N}$ such that $G \cap N \subseteq \bigoplus_{n<n_{0}} G_{n}$. Now, every proper closed subgroup of $\mathbb{T}$ is finite and contained in $\mathbb{Q} / \mathbb{Z}$. Thus, if $\pi_{n}$ denotes the $n$ th-projection of $G$ onto $G_{n}$, we have that either $\pi_{n}(G \cap N)=\mathbb{T}$ or $\pi_{n}(G \cap N) \subseteq \mathbb{Q} / \mathbb{Z}$. If $\operatorname{Ker} \pi_{1}$ were trivial, then $\pi_{1}$ is 1 -to-1 and, therefore, $\pi_{1}(G \cap N)$ contains a finite subgroup $F$ that is isomorphic to its inverse image $\pi_{1}^{-1}(F) \subseteq H$. Thus, we may assume that $\operatorname{Ker} \pi_{1}$ is nontrivial. Then we replace $G \cap N$ by Ker $\pi_{1}$. Applying induction, it follows that $H \cap N \neq\{0\}$. (6) Let $N$ be a compact metrizable subgroup of $b H$ such that $H \cap N=\{0\}$ and let $A \subseteq H$ such that $A+N$ is compact in $b H$. By (2) and (4) we have that $A+(G \cap N)$ is compact in $G$ and, by (5), $G \cap N=\{0\}$. Thus $A=A+(G \cap N)$ is compact in $G$. Since $A \subseteq H$, we obtain that $A$ is compact in $H$. In other words, the group $N$ respects compactness in $H$ if $H \cap N=\{0\}$. (7) Take $N=G_{1}$ and $A=H_{1}$. If $\phi: b H \longrightarrow b H / N$ denotes the canonical quotient map, we have that $\phi(A)=\{0\}$ is trivially compact in $b H / N$. On the other hand, $A+(N \cap H)=H_{1}$, which is not compact in $H$. Therefore $H$ does not strongly respects compactness.

\section{CONClusions AND FinAl REMARKS}

Consider the class of $a \mathcal{B}$ consisting of the totally bounded groups $(H, \mathrm{w})$ such that there exists a locally compact non-compact group $(G, \tau)$ such that (a) $H$ is a subgroup of $G$, (b) $H$ is dense in $(G, \tau)$, and $(\mathrm{c})\left(H, \tau^{+}\right)=(H, \mathrm{w})$. For example, $\mathbb{T} \in \mathcal{B}$ but not in $a \mathcal{B}$, and $\mathbb{Q}^{+} \in a \mathcal{B}$ but not in $\mathcal{B}$. Let us call members of the class $a \mathcal{B}$ almost-Bohr groups. The reason for requiring $\tau$ above to be non-compact is that otherwise, every totally bounded group, being contained in its Weil-completion, would be almost-Bohr. We see then that no totally bounded metrizable group is almost-Bohr, and they are in $\mathcal{B}$ if and only if they are compact (Corollary 3.14).

Problem 6.1. Characterize the class of almost-Bohr groups.

Similarly, consider the classs $k \mathcal{B}$ (resp. $s \mathcal{B}$ ) consisting of the abelian totally bounded groups $(G, \mathrm{w})$ such that there exists a locally compact topology $\tau$ such that $\tau=k_{g}[\mathrm{w}]$ (resp. $\tau=s_{g}[\mathrm{w}]$ ). Obviously, $\mathcal{B} \subseteq k \mathcal{B} \cap s \mathcal{B}$, but for example, the group $G$ in Example 4.16 belongs to $k \mathcal{B} \cap s \mathcal{B}$ but not to $\mathcal{B}$. Similarly, $\mathbb{Q}^{+}$does not belong either to $k \mathcal{B} \cap s \mathcal{B}$, or to $s \mathcal{B}$. Again, we see that no totally bounded metrizable non-compact group is in $k \mathcal{B} \cap s \mathcal{B}$ (Corollary 3.14). When restricting ourselves to non-Ulam measurable cardinals, obviously $k \mathcal{B} \subseteq s \mathcal{B}$.

Problem 6.2. Characterize the class $k \mathcal{B}$ (resp. sB).

Problem 6.3. What is the relation between the classes $k \mathcal{B}$ and $s \mathcal{B}$ ?

More generally, replace the locally compact requirement on the topology $\tau$ in the definitions of the classes $\mathcal{B}, a \mathcal{B}, k \mathcal{B}$, and $s \mathcal{B}$ and request instead that $\tau$ satisfies $\tau^{+} \neq \tau$, to obtain the new classes $\mathcal{B}^{\prime}, a \mathcal{B}^{\prime}, k \mathcal{B}^{\prime}$, and $s \mathcal{B}^{\prime}$. Notice then that compact groups belong to each of $\mathcal{B}, k \mathcal{B}$, and $s \mathcal{B}$ but to none of $\mathcal{B}^{\prime}, a \mathcal{B}, a \mathcal{B}^{\prime}, k \mathcal{B}^{\prime}$, and $s \mathcal{B}^{\prime}$. Since the free abelian group $A(X)$ of any Tychonoff space $X$ respects compactness [9] (4.20), it 
follows that groups of the form $A(X)^{+}$with $X$ infinite belong to each of the classes $\mathcal{B}^{\prime}, a \mathcal{B}^{\prime}, k \mathcal{B}^{\prime}$, and $s \mathcal{B}^{\prime}$, but to none of $\mathcal{B}, a \mathcal{B}, k \mathcal{B}$, or $s \mathcal{B}$. Obviously, $\mathcal{B}^{\prime} \subseteq k \mathcal{B}^{\prime} \cap s \mathcal{B}^{\prime}$, but since semi-Montel spaces and $\mathcal{L}_{\infty}$ groups strongly respect compactness [9] (4.15\&4.16), we see that there are groups belonging to $k \mathcal{B}^{\prime} \cap s \mathcal{B}^{\prime}$ but not to $\mathcal{B}^{\prime}$. Moreover, any dense proper subgroup $H$ of $A(X)^{+}$yields a group in $a \mathcal{B}^{\prime}$ but not in $\mathcal{B}^{\prime}$.

Problem 6.4. Characterize the classes $\mathcal{B}^{\prime}, a \mathcal{B}^{\prime}, k \mathcal{B}^{\prime}$, and $s \mathcal{B}^{\prime}$.

Problem 6.5. What are the relations between the classes in Problem 6.4?

\section{REFERENCES}

1. R. Arens, A topology for spaces of transformations, Ann. Math. 47 (1946), 480-495.

2. A. Arhangel'skii and M. Tkachenko, Topological Groups and Related Structures, vol. 1, 2008, Atlantic Press, AmsterdamParis.

3. W. W. Comfort, Topological groups, Handbook of Set-Theoretic Topology (K. Kunen and J. E. Vaughan, eds.), Elsevier Science Publishers, B. V., Amsterdam, 1984, pp. 1143-1263.

4. W. W. Comfort and D. Remus, Compact groups of Ulam-measurable cardinality: partial converses to theorems of Arhangel'skiน and Varopoulos, Math. Japon. 39 (1994), no. 2, 203-210.

5. W. W. Comfort and K. A. Ross, Topologies induced by groups of characters, Fundamenta Mathematicae 55 (1964), no. 3, 283-291.

6. W. W. Comfort, F. J. Trigos-Arrieta, and T.-S. Wu, The Bohr compactification, modulo a metrizable subgroup, Fund. Math. 143 (1993), 119-136.

7. M. V. Ferrer, S. Hernández, and L. Tárrega, Interpolation sets in spaces of continuous metric-valued functions, J. Math. Analysis and Applications 466 (2018), no. 2, 1426-1442.

8. J. Galindo, Totally bounded group topologies that are Bohr topologies of LCA groups, Topology Proceedings 28(2) (2004), 467-478.

9. J. Galindo and S. Hernández, The concept of boundedness and the Bohr compactification of a MAP Abelian group, Fund. Math. 159 (1999), 195-218.

10. J. Galindo, S. Hernández, and Ta-Sun Wu, Recent results and open questions relating Chu duality and Bohr compactifications of locally compact groups, Open problems in topology. II. Edited by: Elliott Pearl. Amsterdam: Elsevier (2007).

11. I. Glicksberg, Uniform boundedness for groups, Canadian J. Math. 14 (1962), 269-276.

12. S. Hernández, The Dimension of an LCA group in its Bohr Topology, Topology and its Applications 86 (1998), 63-67.

13. S. Hernández and F. J. Trigos-Arrieta, Group duality with the topology of precompact convergence, J. Math. Analysis and Applications 303 (2005), 274-287.

14. E. Hewitt and K. A. Ross, Abstract Harmonic Analysis: Volume I, Structure of Topological Groups, Integration Theory, Group Representations, Grundlehren der mathematischen Wissenschaften, Springer New York, 1963.

15. H. Heyer, Dualität lokalkompakter Gruppen, Springer-Verlag, Berlin-Heidelberg-New York, 1970.

16. R Hughes, Compactness in locally compact groups, Bulletin of the American Mathematical Society 79 (1973), 122-123.

17. N. Noble, k-groups and duality, Trans. Amer. Math. Soc. 151 (1970), 551-561.

18. S. U. Raczkowski and F. J. Trigos-Arrieta, Duality of Totally Bounded Groups, Boletín de la Sociedad Matemática Mexicana 7 (2001), 1-12.

19. G. A. Reid, On sequential convergence in groups, Math. Zeitschrift 102 (1967), 225-235.

20. Francisco Javier Trigos-Arrieta, Pseudocompactness on groups, Ph.D Thesis, Wesleyan University (1991).

21. N. Th. Varopoulos, A theorem on the continuity of homomorphisms of locally compact groups, Proc. Cambridge Phil. Soc. 60 (1964), 449-463.

Departament de Matemàtiques, Universitat Jaume I, 12071 Castelló, Spain.

E-mail address: hernande@uji.es

Department of Mathematics, California State University, Bakersfield, Bakersfield, CA 93311

E-mail address: jtrigos@csub.edu 